\title{
BORDISM INVARIANCE IN KK-THEORY
}

\author{
MICHEL HILSUM
}

\begin{abstract}
A bordism invariance property in bivariant K-theory for unbounded Hilbert modules is proved. Various geometric corollaries are deduced.
\end{abstract}

\section{Introduction}

In a recent work, W. Lück et T. Schick [15, Remark 1.7] asked if the $L^{2}$-index of a twisted signature operator over a Lipschitz manifold is a bordism invariant.

Bordism invariant of the index of elliptic differential operators goes back to the Atiyah-Singer index theorem [16]. Here the method relies on the use of the Calderon projector, and entails pseudodifferential calculus. For the signature operator on a Lipschitz manifold, bordism invariance was shown by N. Teleman [18] by surgery methods. Besides, in K-homology, J. Rosenberg et S. Weinberger [17] have shown analytically the existence of a morphism for a finite cellular complex $X$.

$$
\Omega(X) \rightarrow K_{0}(X)
$$

By using topological methods, C. Carvalho [8], [9] has proved the bordism invariant of the index for families of elliptic differential operators.

In [13], the notion of Hilbert module with odd boundary over a $\mathrm{C}^{*}$-algebra $\mathscr{B}$ has been introduced. It has been shown that the index of such a module with target the topological K-theory group of $\mathscr{B}$ is null.

Here, we propose a general theorem in G. Kasparov's bivariant K-theory which recovers all these cases, and moreover we give a simple proof, although not elementary. Actually, analytical bordism invariance is a mere translation of the simple fact that the $\mathrm{C}^{*}$-algebra $\left.\left.C_{0}(] 0,1\right]\right)$ is K-contractile, and thus, that for any $\mathrm{C}^{*}$-algebras $\mathscr{A}, \mathscr{B}$, the bivariant $\mathrm{K}$-theory group $\left.\left.K K\left(C_{0}(] 0,1\right]\right) \otimes \mathscr{A}, \mathscr{B}\right)$ is trivial.

We start with two $C^{*}$-algebras $\mathscr{A}, \mathscr{B}$, and $(\mathscr{E}, A, \tau)$ a cycle of bivariant $\mathrm{K}$-theory. Assuming that it is a boundary, we prove that its class in $K K(\mathscr{A}, \mathscr{B})$ is null (Theorem 6.2).

Received 11 February 2009. 
Here we use unbounded picture of cycles in G. Kasparov's theory as expounded by S. Baaj and P. Julg [3]. Doing so, we are lead to understand how a symmetric but not self-adjoint operator may define an element in $K K(\mathscr{A}, \mathscr{B})$. Such a question have already been adressed by P. Baum, R. Douglas and M. Taylor [4] when $\mathscr{A}$ is commutative, and $T$ is an order one elliptic differential operator on some non-compact manifold. There, the proof uses the finite speed velocity property of $T$. Here we prove an analogous result, but we relax this latter hypothesis.

As a consequence, we give a positive answer to the question mentioned initially.

Then we will prove a $G$-equivariant version of the map (1.1), where $G$ is a compact Lie group in Corollary 9.3. This construction is related to pseudodifferential operators transversally elliptic to a smooth action of $G$. A similar result for the topological index has been given by C. Farsi [10].

Finally, we shall use this theorem to formulate a non-commutative conservation of total indices under some cutting and gluing operations on manifolds.

\section{Preliminaries}

Let $\mathscr{B}$ be a $\mathrm{C}^{*}$-algebras, $\mathscr{E}$ a Banach $\mathscr{B}$-module; recall then that $\mathscr{E}$ is said to be a Hilbert bimodule if we have a sesquilinear map $\langle.,\rangle:. \mathscr{E} \times \mathscr{E} \rightarrow \mathscr{B}$ such that for $\xi, \eta \in \mathscr{E}, b \in \mathscr{B}$, one has $\langle\xi, \eta\rangle^{*}=\langle\eta, \xi\rangle,\langle\xi, b \eta\rangle=\langle\xi, \eta\rangle b,\langle\xi, \xi\rangle \geq 0$ and $\langle\xi, \xi\rangle=0$ iff $\xi=0$. Then the map $\xi \rightarrow\|\langle\xi, \xi\rangle\|^{\frac{1}{2}}$ is the norm on $\mathscr{E}$.

Let $T: \mathscr{E} \rightarrow \mathscr{E}$ a densely defined linear map. The adjoint of $T$ is defined for those $\xi \in \mathscr{E}$ such that there exists $\eta \in \mathscr{E}$ satisfying for every $\zeta \in \mathscr{E}$ the equality $\langle T \zeta, \xi\rangle=\langle\zeta, \eta\rangle$ and then $T^{*} \xi=\eta$. In general, $T^{*}$ is a closed operator of $\mathscr{B}$-module, which means that $\left(\operatorname{dom} T^{*}\right) \mathscr{B} \subset \operatorname{dom} T^{*}$ and $T^{*}(\xi b)=\left(T^{*} \xi\right) b$ whenever $(\xi, b) \in\left(\operatorname{dom} T^{*}\right) \times \mathscr{B}$.

Equivalently, one may define $T^{*}$ as follows: the orthogonal of the graph of $T$ in $\mathscr{E} \oplus \mathscr{E}$ is the graph of an operator $S$ of $\mathscr{E}$, as $T$ is densely defined, and we put $T^{*}=-S$.

We say that a densely defined linear operator is adjointable if $T^{*}$ is densely defined. A densely defined operator $T$ such that $T^{*}$ is densely defined is closable, its closure is a $\mathscr{B}$-module operator, and one has $\bar{T}=T^{* *}$.

The operator is said to be symmetric if $\langle T \xi, \eta\rangle=\langle\xi, T \eta\rangle$ for $\xi, \eta \in \operatorname{dom} T$, and selfadjoint if $T=T^{*}$. If $T$ is adjointable and bounded, then $T^{*}$ is bounded and everywhere defined. As usual we denote by $\mathscr{L}(\mathscr{E})$ the $\mathrm{C}^{*}$-algebra of bounded adjointable operators of the $\mathscr{B}$-module $\mathscr{E}$, and by $\mathscr{K}(\mathscr{E})$ the closed ideal of compact operators of $\mathscr{E}$, generated by the rank one operators of the form $\zeta \rightarrow \xi\langle\eta, \zeta\rangle$, for $\xi, \eta \in \mathscr{E}$.

We define $\mathscr{L}(T)$ the subalgebra of $\mathscr{L}(\mathscr{E})$ of operators $a \in \mathscr{L}(\mathscr{E})$ such that 
$a(\operatorname{dom} T) \subset \operatorname{dom} T$ and such that the graded commutator $[T, a]$ extends to an element of $\mathscr{L}(\mathscr{E})$.

Lemma 2.1. For $T$ and $T^{*}$ densely defined on $\mathscr{E}$, and $a \in \mathscr{L}(T)$, one has $a^{*} \in \mathscr{L}\left(T^{*}\right)$, and $[a, T]^{*}$ is the closure of $\left[T^{*}, a^{*}\right]$.

Proof. For $\xi \in \operatorname{dom} T, \eta \in \operatorname{dom} T^{*}$, the equality $\left\langle T \xi, a^{*} \eta\right\rangle=\langle[a, T] \xi, \eta\rangle$ $+\left\langle a \xi, T^{*} \eta\right\rangle$ shows that $a^{*} \eta \in \operatorname{dom} T^{*}$ and $\left(T^{*} a^{*}-a^{*} T^{*}\right) \eta=[a, T]^{*} \eta$.

Given a closed linear operator $T$ on $\mathscr{E}$, we shall denote by $\mathscr{W}(T)$ the Hilbert B-module dom $T$ equipped with the B-product: $\langle\xi, \eta\rangle+\langle T \xi, T \eta\rangle$. Recall that a core or an essential domain for a closed operator $T$ is a dense subspace of $\mathscr{W}(T)$ and that $T$ is said to be regular if $1+T^{*} T$ and $1+T T^{*}$ are surjective operators of $\mathscr{E}$, and in that case the inverse operators $\left(1+T^{*} T\right)^{-1}$ and $\left(1+T T^{*}\right)^{-1}$ belongs to $\mathscr{L}(\mathscr{E})$ [14]. If $T=T^{*}$, then these conditions are equivalent to the surjectivity of $\operatorname{im}(i+T)$. Regularity is also equivalent to the adjointability of the natural continuous injection $\mathscr{W}(T) \rightarrow \mathscr{E}$.

We now specify some terms which will be used in the sequel.

i) An unbounded module over $\mathscr{B}$ is the data of a Hilbert module $\mathscr{E}$ over $\mathscr{B}$ with a regular operator $T$. We shall call $(\mathscr{E}, T, \tau)$ an even unbounded module if $\tau$ is a unitary involution such that $\tau T+T \tau=0$.

ii) A symmetric module over $\mathscr{B}$ is an unbounded module $(\mathscr{E}, T)$ with $T \subset$ $T^{*}$.

iii) A closed module over $\mathscr{B}$ is an unbounded module $(\mathscr{E}, T)$ with $T=T^{*}$ and $(i+T)^{-1} \in \mathscr{K}(\mathscr{E})$.

We end this section with a lemma which resembles [13, Lemma 2.3], but with the hypothesis of selfadjointness dropped off. Let $\mathscr{E}$ an Hilbert $\mathscr{B}$-module, $T$ a regular operator on $\mathscr{E}$ and $f \in \mathscr{L}(T)$ with $f=f^{*}$. The map $u_{f}(\xi)=f \xi$ for $\xi \in \operatorname{dom} T$ defines then a continuous map from $\mathscr{W}(T)$ to itself, commuting with $B$ and of norm less than $\sqrt{\|[T, f]\|^{2}+\|f\|^{2}}$.

Lemma 2.2. The map $u_{f}$ is adjointable and we have, for $\eta \in \operatorname{dom} T$ :

$$
u_{f}^{*} \eta=f^{*} \eta+\left(\left(1+T^{2}\right)^{-1} T^{*}\right)[T, f] \eta+\left(1+T^{2}\right)^{-1}[T, f] T \eta .
$$

Proof. The proof is similar to that of [13, Lemma 2.3].

REMARK 2.3. If we assume in the previous lemma that $T \subset T^{*}$, then the same conclusion holds with arbitrary $f \in \mathscr{L}(T)$, as in that case $f^{*} \in$ $\mathscr{L}\left(T^{*}\right) \subset \mathscr{L}(T)$.

Remark 2.4. This lemma will be applied in the following case: Let $T_{i}$ be a regular operator on $\mathscr{E}_{i}$ for $i=1,2$, and $f \in \mathscr{L}\left(\mathscr{E}_{1}, \mathscr{E}_{2}\right)$ such that $f \operatorname{dom} T_{1} \subset$ 
$\operatorname{dom} T_{2}, f^{*} \operatorname{dom} T_{2} \subset \operatorname{dom} T_{1}$, and such that $f T_{1}-T_{2} f$, and $f^{*} T_{2}-T_{2} f^{*}$ are bounded. Then the map $\xi \rightarrow f \xi$ from $\mathscr{W}\left(T_{1}\right)$ to $\mathscr{W}\left(T_{2}\right)$ is bounded and adjointable. This follows from the lemma by taking the direct sum of $T_{1}$ and $T_{2}$.

Still, the same result remains valid with $T_{2}$ singular and $T_{2}^{*}$ densely defined, as in [13, lemma 2.3].

\subsection{Continuous fields of symmetric operators}

Let $\mathscr{E}=\left(\mathscr{E}_{z}\right)_{z \in Z}$ be a $C^{*}$-module over $C(Z) \otimes \mathscr{A}$, in other words a continuous field over $Z$ of Hilbert modules over $\mathscr{A}$. Let $T_{z}$ be a family of symmetric regular operators on $\mathscr{E}_{Z}$. When does this family gives a symmetric regular operator on $\mathscr{E}$ ? Let $T$ acting on $\mathscr{E}$ the domain of which contains those continuous sections $\xi=\left(\xi_{z}\right)$ of $\mathscr{E}$ such that $\left(T_{z} \xi_{z}\right)$ is a section of $\mathscr{E}$. Then $T$ is evidently symmetric and closable

Lemma 2.5. Suppose that under the evaluation maps $e_{z}: \mathscr{E} \rightarrow \mathscr{E}_{z}$, the subspace $e_{z}(\operatorname{dom} T)$ and respectively $e_{z}\left(\operatorname{dom} T^{*}\right)$ are essential domains for $T_{z}$ and respectively $T_{z}^{*}$. Then $T$ is a regular operator on $\mathscr{E}$.

Proof. Let the $2 \times 2$-matrix:

$$
\mathscr{T}=\left(\begin{array}{cc}
0 & T \\
T^{*} & 0
\end{array}\right)
$$

Then the result follow from [11] applied to $\mathscr{T}$.

\section{Half-closed cycles in bivariant K-theory}

Usually, the bivariant K-theory of G. Kasparov is defined by cycles ( $\mathscr{H}, F, \tau)$ where $\mathscr{H}$ is a Hilbert module, $F$ a bounded adjointable operator, and $\tau$ a unitary involution anticommuting with $F$.

S. Baaj and P. Julg [3] have shown how to define a cycle in bivariant K-theory starting with a closed module, that is to say by using unbounded operators instead of bounded ones. This theory is well suited for the study of geometric elliptic differential operators on compact manifolds, as an elliptic pseudodiffernetial operator is essentially self-adjoint. This does not necessarily remains true on non-compact manifolds, and here we give a slight generalisation of this theory by using only symmetric operators.

On non-compact manifolds, cycles in analytical K-homology coming from symmetric elliptic differential operators have been studied by P. Baum, R. Douglas and M. Taylor [4]. The main result obtained there is that, when the operator has order one, then any closed symmetric extension determines a KKcycle. The method uses the notorious "finite speed velocity property". Here 
we show that this condition is not necessary, and that one can obtain under weaker conditions a similar result in a more general framework.

Let $\mathscr{A}, \mathscr{B}$ be two $\mathrm{C}^{*}$-algebras and $\mathscr{E}$ be an $(\mathscr{A}, \mathscr{B})$ Hilbert bimodule, and $T$ a symmetric regular operator on $\mathscr{E}$, such that there is a dense involutive subalgebra $\mathscr{A}_{0} \subset \mathscr{A}$ enjoying the following properties:

(1) For $a \in \mathscr{A}_{0}$, one has $a\left(\operatorname{dom} T^{*}\right) \subset \operatorname{dom} T$.

(2) $\mathscr{A}_{0} \subset \mathscr{L}(T)$.

(3) For $a \in \mathscr{A}$, the operator $\left(1+T^{*} T\right)^{-1} a$ is a compact morphism of $\mathscr{E}$.

Such an operator will be called half-closed in the sequel. In the following lemma, $\hat{T}$ is not necessarily symmetric:

Lemma 3.1. Let $T \subset \hat{T} \subset T^{*}$ be an extension of $T, F=T\left(1+T^{*} T\right)^{-\frac{1}{2}}$ and $\hat{F}=\hat{T}\left(1+\hat{T}^{*} \hat{T}\right)^{-\frac{1}{2}}$. Then, for $a \in \mathscr{A}$, one has $\left(F^{*}-\hat{F}\right) a \in \mathscr{K}(\mathscr{E})$

Proof. Let $\lambda>0, a \in \mathscr{A}_{0}$ and $R_{\lambda}=\left(T^{*}\left(\lambda+T T^{*}\right)^{-1}-\left(\lambda+\hat{T} \hat{T}^{*}\right)^{-1} \hat{T}\right) a$. Then one has:

$$
\begin{aligned}
R_{\lambda}= & \left(\left(\lambda+\hat{T} \hat{T}^{*}\right)^{-1} \hat{T} \hat{T}^{*}\right)\left(T^{*}\left(\lambda+T T^{*}\right)^{-1}\right) a \\
& -\left(\left(\lambda+\hat{T} \hat{T}^{*}\right)^{-1} \hat{T}\right)\left(T T^{*}\left(\lambda+T T^{*}\right)^{-1}\right) a \\
& +\lambda\left(\left(\lambda+\hat{T} \hat{T}^{*}\right)^{-1} \hat{T}\right)\left(\lambda+T T^{*}\right)^{-1} a \\
& -\lambda\left(\lambda+\hat{T} \hat{T}^{*}\right)^{-1}\left(T^{*}\left(\lambda+T T^{*}\right)^{-1}\right) a
\end{aligned}
$$

Here we adopt the rule that when an algebraic expression in under parenthesis or bracket, as for example $\left(T^{*}\left(\lambda+T T^{*}\right)^{-1}\right)$, it means that we consider the closure of the operator. Otherwise, products are meant to be algebraic: for example, $T_{1} T_{2}$ is the operator with domain $T_{2}^{-1}$ (dom $T_{1}$ ). The left side of the equality has four terms which we analyse now. For the first two ones, we remark that if $\zeta \in \operatorname{dom} T$, then $\hat{T}^{*} \zeta=T \zeta$, and $\left(\left(\lambda+\hat{T} \hat{T}^{*}\right)^{-1} \hat{T} \hat{T}^{*}\right) \zeta=$ $\left(\left(\lambda+\hat{T} \hat{T}^{*}\right)^{-1} \hat{T}\right) T \zeta$. As $\operatorname{im}\left(T^{*}\left(\lambda+T T^{*}\right)^{-1}\right) \subset$ dom $T$, the first term becomes equal to $\left(\left(\lambda+\hat{T} \hat{T}^{*}\right)^{-1} \hat{T}\right) T\left(T^{*}\left(\lambda+T T^{*}\right)^{-1}\right) a=\left(\left(\lambda+\hat{T} \hat{T}^{*}\right)^{-1} \hat{T}\right)\left(T T^{*}(\lambda+\right.$ $\left.\left.T T^{*}\right)^{-1}\right) a$, which is the opposite of the second term. Thus the two first terms collapse, and $R_{\lambda}$ reduces to:

$$
R_{\lambda}=\lambda\left(\left(\left(\lambda+\hat{T} \hat{T}^{*}\right)^{-1} \hat{T}\right)\left(\lambda+T T^{*}\right)^{-1}-\left(\lambda+\hat{T} \hat{T}^{*}\right)^{-1}\left(T^{*}\left(\lambda+T T^{*}\right)^{-1}\right)\right) a
$$

As for $\xi \in \operatorname{dom} T^{*}$, one has $\left(\hat{T}-T^{*}\right) a \xi=0$ and as $\operatorname{im}\left(\lambda+T T^{*}\right)^{-1} \subset$ $\operatorname{dom} T^{*}$, one has:

$$
R_{\lambda}=\lambda\left(\left(\left(\lambda+\hat{T} \hat{T}^{*}\right)^{-1} \hat{T}\right)\left[\left(\lambda+T T^{*}\right)^{-1}, a\right]-\left(\lambda+\hat{T} \hat{T}^{*}\right)^{-1}\left(T^{*}\left[\left(\lambda+T T^{*}\right)^{-1}, a\right]\right)\right)
$$


Observe that $\operatorname{im}\left[\left(\lambda+T T^{*}\right)^{-1}, a\right] \subset \operatorname{dom} T^{*}$, thus the last expression makes sense as an everywhere defined closed operator, hence bounded. We show now the equality:

$$
\begin{aligned}
{\left[\left(\lambda+T T^{*}\right)^{-1}, a\right]=} & \left(\lambda+T T^{*}\right)^{-1}[T, a]\left(T^{*}\left(\lambda+T T^{*}\right)^{-1}\right) \\
& +\left(\left(\lambda+T T^{*}\right)^{-1} T\right)\left[T^{*}, a\right]\left(\lambda+T T^{*}\right)^{-1}
\end{aligned}
$$

Here $[T, a],\left[T^{*}, a\right]$ means the bounded and closable operator defined on $\operatorname{dom} T$ and $\operatorname{dom} T^{*}$, and the expressions on the r.h.s. are defined upon all of $\mathscr{E}$. This equality would be obvious if all the operators were bounded. However, for example in the second term of the r.h.s, it is not possible to write the equality $\left(\left(\lambda+T T^{*}\right)^{-1} T\right)\left[T^{*}, a\right]\left(\lambda+T T^{*}\right)^{-1}=\left(\lambda+T T^{*}\right)^{-1} T\left[T^{*}, a\right]\left(\lambda+T T^{*}\right)^{-1}$, as $\operatorname{im}\left[T^{*}, a\right]\left(\lambda+T T^{*}\right)^{-1}$ is not necessarily included in $\operatorname{dom} T$. To proceed, observe that:

$$
\begin{aligned}
{\left[\left(\lambda+T T^{*}\right)^{-1}, a\right]=} & \left(\left(\lambda+T T^{*}\right)^{-1} T T^{*}\right) a\left(\lambda+T T^{*}\right)^{-1} \\
& -\left(\lambda+T T^{*}\right)^{-1} a T T^{*}\left(\lambda+T T^{*}\right)^{-1}
\end{aligned}
$$

and that:

$$
\begin{aligned}
\left(\left(\lambda+T T^{*}\right)^{-1} T T^{*}\right) a\left(\lambda+T T^{*}\right)^{-1}= & \left(\left(\lambda+T T^{*}\right)^{-1} T\right) T^{*} a\left(\lambda+T T^{*}\right)^{-1} \\
= & \left(\left(\lambda+T T^{*}\right)^{-1} T\right)\left[T^{*}, a\right]\left(\lambda+T T^{*}\right)^{-1} \\
& +\left(\left(\lambda+T T^{*}\right)^{-1} T\right) a T^{*}\left(\lambda+T T^{*}\right)^{-1} \\
= & \left(\left(\lambda+T T^{*}\right)^{-1} T\right)\left[T^{*}, a\right]\left(\lambda+T T^{*}\right) \\
& +\left(\lambda+T T^{*}\right)^{-1} T a T^{*}\left(\lambda+T T^{*}\right)^{-1} \\
= & \left(\left(\lambda+T T^{*}\right)^{-1} T\right)\left[T^{*}, a\right]\left(\lambda+T T^{*}\right)^{-1} \\
& +\left(\lambda+T T^{*}\right)^{-1}[T, a] T^{*}\left(\lambda+T T^{*}\right)^{-1} \\
& +\left(\lambda+T T^{*}\right)^{-1} a T T^{*}\left(\lambda+T T^{*}\right)^{-1}
\end{aligned}
$$

All these equality are valid upon all of $\mathscr{E}$, that is to say for every $\xi \in \mathscr{E}$. For example, $\left(\lambda+T T^{*}\right)^{-1} \operatorname{TaT}^{*}\left(\lambda+T T^{*}\right)^{-1}$ is defined for all $\xi \in \mathscr{E}$, as $\left.T^{*}\left(\lambda+T T^{*}\right)^{-1}\right) \xi \in \operatorname{dom} T$, and $a(\operatorname{dom} T) \subset \operatorname{dom} T$. The two terms of the r.h.s of equation (3.2) is a bounded operator the image of which is contained in $\operatorname{dom} T^{*}$; thus we get:

$$
\begin{aligned}
R_{\lambda}= & \lambda\left(\left(\lambda+\hat{T} \hat{T}^{*}\right)^{-1} \hat{T}\right)\left(\lambda+T T^{*}\right)^{-1}[T, a]\left(T^{*}\left(\lambda+T T^{*}\right)^{-1}\right) \\
& \left.+\lambda\left(\left(\lambda+\hat{T} \hat{T}^{*}\right)^{-1} \hat{T}\right)\left(\lambda+T T^{*}\right)^{-1} T\right)\left[T^{*}, a\right]\left(\lambda+T T^{*}\right)^{-1} \\
& -\lambda\left(\lambda+\hat{T} \hat{T}^{*}\right)^{-1}\left(T^{*}\left(\lambda+T T^{*}\right)^{-1}\right)[T, a]\left(T^{*}\left(\lambda+T T^{*}\right)^{-1}\right) \\
& +\lambda\left(\lambda+\hat{T} \hat{T}^{*}\right)^{-1}\left(\left(\lambda+T T^{*}\right)^{-1} T\right)\left[T^{*}, a\right]\left(\lambda+T T^{*}\right)^{-1}
\end{aligned}
$$


Each of the four term in the right hand side of the later is a compact morphism the norm of which is a $\mathcal{O}\left(\lambda^{-1}\right)$ as $\lambda$ goes to $+\infty$, and norm continuous w.r.t. $\lambda$.

The classic formula gives us that:

$$
\left(F^{*}-\hat{F}\right) a=\frac{1}{\pi} \int_{1}^{+\infty} R_{\lambda} \frac{d \lambda}{\sqrt{\lambda-1}}
$$

which is therefore compact.

THEOREM 3.2. Let $T$ a symmetric regular operator on $\mathscr{E}$ enjoying the properties (1)-(3) above, $T \subset \hat{T} \subset T^{*}$ be an extension and $\tau$ a unitary involution anticommuting with $T$ and $\hat{T}$, and $\hat{F}=\hat{T}\left(1+\hat{T}^{*} \hat{T}\right)^{-\frac{1}{2}}$. Then for $a \in \mathscr{A}$, the operators $\left(\hat{F}^{*}-\hat{F}\right) a,\left(\hat{F}^{2}-1\right)$ a are compact morphisms of $\mathscr{E}$ and thus $(\mathscr{E}, \hat{F}, \tau)$ is a bounded cycle of bivariant $K$-theory. Moreover, the class of $(\mathscr{E}, F, \tau)$ in $K K(\mathscr{A}, \mathscr{B})$ does not depend of the extension's choice.

Proof. The compactness of $\left(\hat{F}^{*}-\hat{F}\right) a=\left(\hat{F}^{*}-F^{*}\right) a+\left(F^{*}-\hat{F}\right) a$ results from the preceding lemma. To prove compactness of $\left(\hat{F}^{2}-1\right) a=$ $\left(1+\hat{T}^{*} \hat{T}\right)^{-1} a$, take $b, c \in \mathscr{A}_{0}$; let $I$ (resp. $\left.\hat{I}\right)$ be the injection of $\mathscr{W}(T)$ (resp. $\mathscr{W}(\hat{T}))$ in $\mathscr{E}$, which is bounded adjointable. Then by Lemma 2.2 and Remark 2.4, the multiplication operator by $c$ from $\mathscr{W}(\hat{T})$ to $\mathscr{W}(T)$ is bounded adjointable, and the composition $b \circ I \circ c \circ \hat{I}^{*}$ is a compact morphism as $b \circ I$ is, and therefore $\left(1+\hat{T}^{*} \hat{T}\right)^{-1} b c$ is compact, and the assertion follows as $\mathscr{A}_{0}$ and thus $\mathscr{A}_{0}^{2}$ are dense in $\mathscr{A}$.

As in particular for any $a \in \mathscr{A}$ the operator $(\hat{F}-F) a$ is compact, it shows that $(\mathscr{E}, \hat{F}, \tau)$ is equal to the class of $(\mathscr{E}, F, \tau)$.

\subsection{External tensor product}

As an application, we may characterize the external intersection product of two such operators. Let $\left(\mathscr{E}_{i}, T_{i}, \tau_{i}\right)$ be two half-closed operator on $\left(\mathscr{A}_{i}, \mathscr{B}_{i}\right)$. Then $T_{1} \hat{\otimes} 1+1 \hat{\otimes} T_{2}$ is a regular symmetric operator on $\mathscr{E}_{1} \hat{\otimes} \mathscr{E} 2$. Then the following may be proved exactly as in [3, Théorème 3.2].

Lemma 3.3. The external tensor product in $K K\left(\mathscr{A}_{1} \otimes \mathscr{A}_{2}, \mathscr{B}_{1} \otimes \mathscr{B}_{2}\right)$ is the class defined by $\left(\mathscr{E}_{1} \hat{\otimes} \mathscr{E}_{2}, T_{1} \hat{\otimes} 1+1 \hat{\otimes} T_{2}\right)$.

\subsection{Naturality with respect to restriction}

Another interesting consequence is the following: let $(\mathscr{E}, T)$ as above and $\phi: \mathscr{A}_{1} \rightarrow \mathscr{A}$ be a morphism such that $\phi^{-1}\left(\mathscr{A}_{0}\right)$ is dense in $\mathscr{A}_{1}$. Suppose that the restriction $T_{1}$ of $T$ to $\mathscr{E}_{1}$, the closure of $\phi\left(\mathscr{A}_{1}\right) \mathscr{E}$, is regular. Then $\left(\mathscr{E}_{1}, T_{1}\right)$ defines an element of $K K^{1}\left(\mathscr{A}_{1}, \mathscr{B}\right)$ which is the image of $[\mathscr{E}, T]$ under $\phi_{*}$. 


\subsection{Riemannian incomplete manifolds}

Let $(V, g)$ be a Riemannian manifold, and $D$ be a Dirac type operator acting upon some Clifford module bundle $S$ on $V$. Then it is well known that when the Riemannian metric structure is complete, then $D$ is a selfadjoint operator with locally compact resolvent, and thus gives a K-homology class of $V$.

What does happen when the structure is incomplete? Then $D$ is a halfclosed operator over $C_{0}(V)$, and by the device described above, one gets again a K-homology class of $V$. The operator $F$ considered in the lemma above is exactly the same that is usually defined in a bounded framework.

Moreover, by the remark above, for any open $U \subset V$, the restriction of $D$ to $U$ determine an element in $K^{*}\left(C_{0}(U)\right)$ equal to the image of $[D]$ under the inclusion $U \rightarrow V$. However, it is not so clear that the element obtained in independant of the metric. Let $g_{t}$ be a path of Riemannian metrics on $V$, uniformly continuous on every compact subset of $V$, and suppose that $S$ is a Clifford module on $V \times[0,1]$ for this metric. Let $D=\left(D_{t}\right)$ the family of Dirac type operators. Then one can deduce from Lemma 2.5 that $D$ is regular.

Lemma 3.4. With the hypothesis above, $D_{0}$ and $D_{1}$ give the same class in $K_{0}(V)$.

Proof. This a simple consequence of Theorem 3.2: $D$ determines an element of $K_{0}(C(V) \otimes C([0,1])$.

Example 3.5. Let $V=] 0,1\left[\right.$ with the standard Riemannian metric $d t^{2}$, and $A$ be the closure of the operator $-i \frac{\partial}{\partial t}$ on $C_{c}^{\infty}(] 0,1[)$ in $L^{2}([0,1])$. Then the element of $K_{1}(] 0,1[)=K K^{1}\left(C_{0}(] 0,1[), \mathrm{C}\right)$ defined by $\left(L^{2}([0,1]), A\right)$ is the so-called dual Dirac or fundamental class, generator of $K_{1}(] 0,1[)=\mathbf{Z}$.

\section{Axioms of unbounded cycles: a counter-example}

Classically, axioms of unbounded module entails the condition $a(\operatorname{dom} D) \subset$ dom $D$, and $[D, a]$ bounded for $a$ belonging to a dense *-algebra of $\mathscr{A}[3]$. In the second edition of B. Blackadars' book [6, page 163], the condition $a(\operatorname{dom} D) \subset \operatorname{dom} D$ is substituted with the space of $\xi \in \operatorname{dom} D$ such that $a \xi \in \operatorname{dom} D$ is dense in $\mathscr{E}$.

We show here that this last condition is not valid, by exhibiting a counterexample.

Let $V$ be an even dimensional smooth oriented manifold with boundary $W$. Then, with a Riemannian metric collared near the boundary, the signature operator $D_{0}$ acts on the Hilbert space $\mathscr{E}$ of square integrable differential forms, with essential domain the space of differential forms compactly supported in $V-W$; it satisfies $D_{0} \subset D_{0}^{*}$, is not self-adjoint $D_{0} \neq D_{0}^{*}$ and $a \in \mathscr{L}\left(D_{0}\right)$ for $a \in C_{\infty}(V)$ the space of smooth functions up to the boundary on $V$. Among 
the self-adjoint extensions of $D_{0}$, there is a remarkable one $D$, associated to the positive part of the spectrum of the signature operator on $W$, and studied first by Atiyah-Patodi-Singer [1]. Then $D$ satisfies these properties: $\left(1+D^{2}\right)^{-1}$ is compact, and for $a \in C_{\infty}(V)$, so that $a$ is smooth up to the boundary, then the space of $\xi \in \operatorname{dom} D$ such that $a \xi \in \operatorname{dom} D$ is dense in $\mathscr{E}$, as it contains dom $D_{0}$, and $[D, a]$ is bounded on that space.

However, $D$ does not define a K-homology class of $C(V)$. Let us assume the contrary and write $z \in K^{0}(C(V))$ for the element defined by $D$ and $z_{0} \in$ $K^{0}\left(C_{0}(V)\right)$ that one be given by $D_{0}$, and let $j: V-W \rightarrow V$ be the inclusion. Then $j^{*}(z)=z_{0}$. Besides, we may find a non trivial $y \in K_{0}\left(C_{0}(V-W)\right)$ such that $j_{*}(y)=0$ and $y \otimes z_{0} \neq 0$ : take for example $x_{0} \in V-W$ and $y$ the pushforward in $K_{0}\left(C_{0}(V-W)\right)$ of a generator of $K^{0}\left(x_{0}\right) \simeq \mathrm{Z}$. Then one would have the nonsense $0=j_{*}(y) \otimes z=y \otimes z_{0} \neq 0$.

\section{Boundary Modules (even dimensional case)}

Let $\left(\mathscr{E}_{b}, A, \tau\right)$ be an even unbounded bimodule over $(\mathscr{A}, \mathscr{B})$, with $A=A^{*}$, and on the external tensor product $\mathscr{E}=\mathscr{E}_{b} \otimes L^{2}(]-\infty,+\infty[)$, let $\Psi(A)$ be the closure of the operator given by the formula:

$$
\Psi(A)=i \tau \otimes 1(1 \otimes \partial+A \otimes 1)
$$

where $\partial f(u)=-\frac{d f}{d u}$ is the usual derivative on the line. The subspace $\mathscr{E}_{b} \otimes$ $C_{c}(]-\infty,+\infty[)$ is an essential domain for $\Psi(A)$ which is a regular selfadjoint operator [12, Lemma 5.1]. Besides, for every $\varphi \in C_{c}(]-\infty,+\infty[), 1 \otimes \varphi(i+$ $\Psi(A))^{-1} \in \mathscr{K}(\mathscr{E})$. Let us now introduce the notion of a boundary $(\mathscr{A}, \mathscr{B})$ module.

Definition 5.1. Let $(\mathscr{E}, T)$ (resp. $\left.\left(\mathscr{E}_{b}, A, \tau\right)\right)$ be a symmetric bimodule (resp. a closed module) over $(\mathscr{A}, \mathscr{B})$. Then $(\mathscr{E} b, A, \tau)$ is the boundary of $(\mathscr{E}, T)$ if there is a projection $p$ in $\mathscr{L}(\mathscr{E})$, commuting with $\mathscr{A}$, an isomorphism of $(\mathscr{A}, \mathscr{B})$ Hilbert bimodule and $\theta: \operatorname{im} p \rightarrow \mathscr{E}_{b} \otimes L^{2}([0,1])$ and enjoying the following properties, where, for $\varphi \in C([0,1]), b(\varphi) \in \mathscr{L}(\mathscr{E})$ is given by $b(\varphi)=\theta^{*}(1 \otimes \varphi) p+\varphi(1)(1-p)$ :

(a) For $\left.\left.\varphi \in C_{c}^{\infty}(] 0,1\right]\right)$, then $b(\varphi) \operatorname{dom} T^{*} \subset \operatorname{dom} T$ and $T^{*} b(\varphi) \xi=$ $T b(\varphi) \xi$ when $\xi \in \operatorname{dom} T^{*}$.

(b) For $\varphi \in C_{c}^{\infty}(] 0,1[)$, then $1 \otimes \varphi(\operatorname{dom} \Psi(A))=\theta \circ b(\varphi)(\operatorname{dom} T)$, and for $\xi \in b(\varphi) \operatorname{dom} T$, one has $\theta T \xi=\Psi(A) \theta \xi$

(c) If $\varphi, \psi \in C^{\infty}([0,1])$ with support $\psi \cap \operatorname{support} \varphi=\emptyset$, then $b(\varphi) T^{*} b(\psi)$ $=0$.

Remark 5.2. Condition (a) implies that, whenever $\left.\varphi \in C_{c}^{\infty}(10,1]\right)$, then $T^{*} b(\varphi)=T b(\varphi)$, that is to say, for every $\xi \in \mathscr{E}$ with $b(\varphi) \xi \in \operatorname{dom} T^{*}$, then 
$b(\varphi) \xi \in \operatorname{dom} T$ and $T^{*} b(\varphi) \xi=T b(\varphi) \xi$. To see this, let $\left.\left.\psi \in C_{c}^{\infty}(] 0,1\right]\right)$ such that $\psi \varphi=\varphi$. Then by (a), as $b(\psi) b(\varphi)=b(\psi \varphi)=b(\varphi)$, then $b(\varphi) \xi=$ $b(\psi) b(\varphi) \xi \in \operatorname{dom} T$, and $T^{*} b(\varphi) \xi=T^{*} b(\psi) b(\varphi) \xi=T b(\psi) b(\varphi) \xi=$ $\operatorname{Tb}(\varphi) \xi$.

Example 5.3. Obviously, we can take $\mathscr{E}=\mathscr{E}_{b} \otimes L^{2}([0,+\infty[)$ with $T$ the closure on $C_{c}(] 0,+\infty$ [) of the matrix given by the formula (5.1), in which case the boundary is given by $\left(\mathscr{E}_{b}, A, \tau\right)$.

With the hypothesis of the last definition, let $b_{0}: L^{\infty}([0,1]) \rightarrow \mathscr{L}(\mathscr{E})$ be the morphism given by $b_{0}(\varphi)=\theta^{*}(1 \otimes \varphi) p \xi$; thus if $\varphi$ is continuous, then $b(\varphi)-b_{0}(\varphi)=\varphi(1)(1-p)$. Then one has:

Lemma 5.4. Let $\varphi$ be a Lipschitz function on $[0,1]$. Then $b(\varphi) \in \mathscr{L}(T)$ and $[T, b(\varphi)]=b_{0}\left(\varphi^{\prime}\right)$, where $\varphi^{\prime}=\frac{d \varphi}{d t}$ is the derivative on the line.

Moreover, if $\varphi(1)=0$, then $b(\varphi)\left(T^{*} T+1\right)^{-1} \in \mathscr{K}(\mathscr{E})$.

Proof. Suppose first that $\varphi$ is smooth and $\operatorname{support}(\varphi) \subset] 0,1[$, and let $\psi \in C_{c}^{\infty}(] 0,1[)$ such that $\psi \varphi=\varphi$. Then by properties $(b)$, (c) of the definition, one has for $\xi \in \operatorname{dom} T,[T, b(\varphi)] \xi=[T, b(\varphi)] b(\psi) \xi=\theta^{-1}[\Psi(A), 1 \otimes$ $\varphi)] 1 \otimes \psi \theta p \xi$. Thus $[T, b(\varphi)]=b_{0}\left(\varphi^{\prime}\right)$ is bounded.

Suppose then $\varphi(0)=\varphi(1)$. Then we may assume that $\varphi(0)=0$ and there exists a sequence $\varphi_{k}$ in $C_{c}^{\infty}(] 0,1[)$ converging to $\varphi$ in the Lipschitz norm. For $\xi \in \operatorname{dom} T$, one has $\lim T \varphi_{k} \xi=\lim \left(\left[T, \varphi_{k}\right]+\varphi_{k} T\right) \xi=\lim b_{0}\left(\varphi_{k}^{\prime}\right) \xi+$ $\lim \varphi_{k} T \xi=b_{0}\left(\varphi^{\prime}\right) \xi+\varphi T \xi$, by the Lebesgue dominated convergence theorem. As $T$ is closed, $T \varphi \xi=\lim T \varphi_{k} \xi$, and thus $[T, \varphi]=b_{0}\left(\varphi^{\prime}\right)$ is bounded.

Suppose then support $\varphi \subset\left[0, \frac{1}{2}[\right.$; then one has $a(\tilde{\varphi}) \in \mathscr{L}(T)$ with $\tilde{\varphi}(t)=$ $\varphi(t)$ on $\left[0, \frac{1}{2}[\right.$ and $\tilde{\varphi}(t)=\varphi(1-t)$ on $\left.] \frac{1}{2}, 0\right]$. Let $\psi$ s.t. $\psi \varphi=\varphi$ and support $\psi \subset\left[0, \frac{1}{2}[\right.$; then for $\xi \in \operatorname{dom} T$ one has, by property (c) of Definition 5.1, $b(\psi)[T, b(\tilde{\varphi})] \xi=b_{0}(\varphi) \xi$ and thus $b(\varphi) \in \mathscr{L}(T)$.

Finally, every $\varphi$ can be written $\varphi=\varphi_{1}+\varphi_{2}$ where support $\varphi_{1} \subset\left[0, \frac{1}{2}[\right.$ and $\varphi_{2}(0)=\varphi_{2}(1)$, and we are done.

To prove that $b(\varphi)\left(T^{*} T+1\right)^{-\frac{1}{2}} \in \mathscr{K}(\mathscr{E})$, it suffices by continuity to take $\varphi \in C_{c}^{\infty}(] 0,1[)$. Let $\mathscr{W}_{0}$ be the closure in $\mathscr{W}(T)$ of $\theta^{-1}\left(\mathscr{E}_{b} \otimes C_{c}^{\infty}(] 0,1[)\right)$; by Definition 5.1(c), the injection $\mathscr{W}_{0} \rightarrow \mathscr{E}$ is compact; otherwise, by Lemma 2.2 and Remark 2.4, the map $\xi \rightarrow b(\varphi) \xi$ from $\mathscr{W}(T)$ to $\mathscr{W}_{0}$ is adjointable. Then compactness of $b(\varphi)\left(T^{*} T+1\right)^{-1}$ follows from the factorisation:

$$
\mathscr{E} \stackrel{\left(T^{*} T+1\right)^{-1}}{\longrightarrow} \mathscr{W}(T) \stackrel{b(\varphi)}{\longrightarrow} \mathscr{W}_{0} \longrightarrow \mathscr{E}
$$

Remark 5.5. It follows from above that $(\mathscr{E}, T)$ comes up with the structure of a $(\mathscr{A} \otimes C([0,1]), \mathscr{B})$-bimodule. 


\section{Boundary invariance}

Let $(\mathscr{E}, T)$ an $\mathscr{A}, \mathscr{B}$-bimodule with boundary $\left(\mathscr{E}_{b}, A, \tau\right)$.

Lemma 6.1. Let $(\mathscr{E}, T)$ with boundary $\left(\mathscr{E}_{b}, A, \tau\right)$. Then the induced element of the bivariant group $K K\left(C_{0}(] 0,1[) \otimes \mathscr{A}, \mathscr{B}\right)$ is the external intersection product of $\left(\mathscr{E}_{b}, A, \tau\right)$ with the fundamental class, generator of $K_{1}\left(C_{0}(] 0,1[)\right)$.

Proof. By the results of the previous sections, the induced element is the external cup-product of $\left(\mathscr{E}_{b}, A, \tau\right)$ by the class of the symmetric operator $-i \frac{\partial}{\partial t}$ on $L^{2}([0,1])$ in $K_{1}\left(C_{0}(] 0,1[)\right)$, which is the Dirac class.

TheOREM 6.2. Let $(\mathscr{E}, T)$ with boundary $\left(\mathscr{E}_{b}, A, \tau\right)$. If a $\left(1+T^{*} T\right)^{-1} \in$ $\mathscr{K}(\mathscr{E})$, for $a \in C(] 0,1]) \otimes \mathscr{A}$, then the class of $\left(\mathscr{E}_{b}, A, \tau\right)$ is zero in $K K(\mathscr{A}, \mathscr{B})$.

Proof. As $a\left(1+T^{*} T\right)^{-1}$ is a compact morphism, and by Lemma 5.4, $(\mathscr{E}, T)$ defines a class in $K K(C(] 0,1]) \otimes \mathscr{A}, \mathscr{B})$. We know that $\left.K K\left(C_{0}(] 0,1\right]\right)$ $\otimes \mathscr{A}, \mathscr{B})=\{0\}$, as $\left.\left.C_{0}(] 0,1\right]\right) \otimes \mathscr{A}$ is a K-contratible $C^{*}$-algebra $[6,19.1]$ and thus the induced class in $K K\left(C_{0}(] 0,1[) \otimes \mathscr{A}, \mathscr{B}\right)$ is null. Now the intersection product by the Dirac element implements an isomorphism of $K K^{1}(\mathscr{A}, \mathscr{B})$ with $K K\left(C_{0}(] 0,1[) \otimes \mathscr{A}, \mathscr{B}\right)$ and so, by the previous lemma, $\left(\mathscr{E}_{b}, A, \tau\right)$ is null.

\section{The odd dimensional case}

Here we state the analogous odd dimensional result. Let $(\mathscr{E}, T, \tau)$ over $(\mathscr{A}, \mathscr{B})$, with boundary $\left(\mathscr{E}_{b}, A\right)$ (cf. [13]). Under the hypothesis which govern the theorem above, we want to show that the class of $\left(\mathscr{E}_{b}, A\right)$ is zero in $K K^{1}(\mathscr{A}, \mathscr{B})$.

Let $C_{1}$ be the complexified Clifford algebra of R with standard euclidean metric; then by definition, $K K^{1}(\mathscr{A}, \mathscr{B}):=K K\left(\mathscr{A}, \mathscr{B} \otimes C_{1}\right)$, and the element associated to $\left(\mathscr{E}_{b}, A\right)$ is the class of $\left(\mathscr{E}_{b} \otimes C^{2}, A \oplus-A, 1 \otimes \tau_{1}\right)$, where $\tau_{1}$ is the involution on $\mathrm{C}^{2}$ giving the grading (it intertwines the two elements of the standard basis). Before stating our next theorem, we recall a lemma.

Lemma 7.1. Let $\mathscr{A}_{i}, \mathscr{B}_{i}$ for $i=1,2$ be $C^{*}$-algebras, and $\left(\mathscr{E}_{i}, A_{i}\right)$ for $i=$ 1,2 closed modules over $\left(\mathscr{A}_{i}, \mathscr{B}_{i}\right)$. Then the external intersection product in $K K\left(\mathscr{A}_{1} \otimes \mathscr{A}_{2}, \mathscr{B}_{1} \otimes \mathscr{B}_{2}\right)$ of the element associated to $\left(\mathscr{E}_{i}, A_{i}\right)$ for $i=1,2$ is represented by the cycle $(\mathscr{E}, A, \tau)$, where $\mathscr{E}=\mathscr{E}_{1} \otimes \mathscr{E}_{2} \otimes \mathrm{C}^{2}$, and:

$$
A=\left(\begin{array}{cc}
0 & -i A_{1} \otimes 1+1 \otimes A_{2} \\
i A_{1} \otimes 1+1 \otimes A_{2} & 0
\end{array}\right)
$$

Proof. This follows from 3.1 above, and basic Clifford algebras properties as expounded in [6] for example.

Then one has then statement analogous to Theorem 6.2: 
TheORem 7.2. Let $(\mathscr{E}, T, \tau)$ with boundary $\left(\mathscr{E}_{b}, A\right)$. If $a\left(1+T^{*} T\right)^{-1} \in$ $\mathscr{K}(\mathscr{E})$, for $a \in C(] 0,1]) \otimes \mathscr{A}$, then the class of $\left(\mathscr{E}_{b}, A\right)$ is zero in $K^{1}(\mathscr{A}, \mathscr{B})$.

Proof. By definition, $(\mathscr{E}, T, \tau)$ on the image of $p$ has the form

$$
\Psi(A)=\left(\begin{array}{cc}
0 & -\frac{\partial}{\partial u}+A \\
\frac{\partial}{\partial u}+A & 0
\end{array}\right)
$$

By the preceding lemma, $\Psi(A)$ acting on $L^{2}([0,1]) \otimes \mathscr{E}_{b} \otimes C^{2}$ with standard grading, is a cycle in $K K\left(C_{0}(] 0,1[) \otimes \mathscr{A}, \mathscr{B}\right)$ which represents the intersection product of $\left[\mathscr{E}_{b}, A\right] \in K K^{1}(\mathscr{A}, \mathscr{B})$ with the Dirac fundamental class of $K K^{1}\left(C_{0}(] 0,1[), C\right)$. Thus $\left[\mathscr{E}_{b}, T, \tau\right]=0$ by Theorem 6.2 .

\section{Signature operators and coverings}

This section is devoted to the question raised by W. Lück and T. Schick. Let $V$ a Lipschitz oriented manifold of even dimension, $E$ a complex hermitian Lipschitz vector bundle over $V, f: V \rightarrow B \Gamma$ a continous map and $\tilde{V}$ the Galois covering associated, which is a Lipschitz manifold.

Given a (measurable) Riemannian metric $g$ on $V$, there is a lifting $\tilde{g}$ to $\tilde{V}$. The group $\Gamma$ acts by unitary operator on $\mathscr{H}=L^{2}\left(V, \Lambda_{\mathrm{C}}\left(T^{*} \tilde{V}\right) \otimes \tilde{E}\right)$, the Hilbert space of square integrable differential forms with coefficient in the pullback $\tilde{E}$ of $E$ to $\tilde{V}$, and let $\mathcal{U}$ be the commutant of this representation, which is a type $I I_{\infty}$ von Neumann algebra coming with a faithful nomal semi-finite trace $\phi$.

Let $\tau$ be the Hodge unitary involution on $\mathscr{H}$. Given a unitary $\Gamma$-invariant connection $\nabla^{E}$ on $\tilde{E}$ ( such a connection may be obtained by pulling back on $\tilde{V}$ a unitary connection on $E$ ), the signature operator $\tilde{A}^{E}=\nabla^{E}-\tau \nabla^{E} \tau$ on $\mathscr{H}$ is a closed self-adjoint operator affiliated to the commutant of the representation of $\Gamma$ on $\mathscr{H}$, and its resolvent is a compact operator of $\mathscr{M}$ in the sense of Breuer, which implies in particular that $\operatorname{ker} \tilde{A}^{E}$ is a $\Gamma$-finite subspace affiliated to $\mathcal{M}$.

Definition 8.1. The $\phi$-index of $\tilde{A}^{E}$ is the difference $\operatorname{Ind}_{\phi}\left(\tilde{A}^{E}\right)=$ $\operatorname{dim}_{\phi}\left(\operatorname{ker} A^{E,+}\right)-\operatorname{dim}_{\phi}\left(\operatorname{ker} A^{E,-}\right)$.

Proposition 8.2. With the notation above, suppose that there exists a Lipschitz oriented manifold $Z$ with boundary $\partial Z=W$, such that $f$ and $E$ extend to $Z$ : then $\operatorname{Ind}_{\phi}\left(\tilde{A}^{E}\right)=0$.

The proof is postponed until later. Actually, we will prove a stronger result, namely the nullity of a certain class in a K-theory group. Let $\mathscr{A}$ be the reduced $\mathrm{C}^{*}$-algebra of $\Gamma$, so that $\mathscr{A}$ is the $\mathrm{C}^{*}$-algebra acting in $l^{2}(\Gamma)$ generated by the left regular representation. Let $\mathscr{H}_{c} \subset \mathscr{H}$ be the subspace of forms with 
compact support, and for $\xi, \eta \in \mathscr{H}_{c}$, defines a finitely supported function on $\Gamma$ by the formula (here $*$ means the Hodge unitary operator):

$$
\langle\xi, \eta\rangle(\gamma)=\int_{\tilde{V}} * \bar{\xi} \wedge\left(\gamma^{*}\right)^{-1}(\eta)
$$

Then $\mathscr{H}_{c}$ with this product is a pre-Hilbert module over $\mathscr{A}$, and its completion $\mathscr{E}_{b}$ is a Hilbert module over $\mathscr{A}$, the action of $a \in \mathrm{C}[\Gamma]$ on $\xi \in \mathscr{H}_{c}$ be given by:

$$
\xi a=\sum_{\gamma} a(\gamma) \gamma^{*} \xi
$$

The following is nowadays classical:

Proposition 8.3. The connection $\nabla^{E}$ extends to a closed regular operator on $\mathscr{E}_{b}$ and the signature operator $A^{E}=\nabla^{E}-\tau \nabla^{E} \tau$ is a selfadjoint operator of $\mathscr{E}_{b}$ with compact resolvent.

Let $M$ be a topological space and $j: V \rightarrow M$ continuous. Thus one obtains a cycle $\left(\mathscr{E}_{b}, A^{E}, \tau\right)$ and a well defined class in $K K(C(M), \mathscr{A})$, denoted $\Sigma^{E}$. Besides, let $Z$ be an oriented Lipschitz manifold with boundary lip-isomorphic to $V$ such $f: V \rightarrow B \Gamma$, and $E \rightarrow V$ have extension to $Z$. Then one has a module $\mathscr{E}$ over $(C(Z), \mathscr{A})$, and a symmetric operator $T$ constructed in a similar way. The fact that $T$ is a regular operator on $\mathscr{E}$ follows from [13, Proposition 3.9], and by considering the double of $Z$, which is a closed manifold. Thus we have shown that $\left(\mathscr{E}_{b}, A, \tau\right)$ is the boundary of $(\mathscr{E}, T)$. We have now readily as a consequence of Theorem 6.2:

THEOREM 8.4. With the notations above, let $Z$ be an oriented Lipschitz manifold with boundary lip-isomorphic to $M$. If $f: V \rightarrow B \Gamma, E \rightarrow M$ and $j: V \rightarrow M$ have extension to $Z$, then $\Sigma^{E}=0$ in $K K(C(M), \mathscr{A})$.

Proof of Proposition 8.2. Take $M$ to the connected zero dimensional manifold, so that $C(M)=\mathrm{C}$. The trace $\phi$ gives on $\mathscr{E}$ a pre-Hilbert space structure by putting $q(\xi, \eta)=\phi(\langle\xi, \eta\rangle)$ for $\xi, \eta \in \mathscr{E}$. The completion of $\mathscr{E}$ w.r.t $q$ is equal to $\mathscr{H}$, and $A^{E}$ gives on $\mathscr{H}$ an essentially selfadjoint operator the closure of which is precisely $\tilde{A}^{E}$. The trace $\phi$ extends to a group homomorphism of $K_{0}(\mathscr{A})$ to $C$ and one has $\operatorname{Ind}_{\phi}\left(\tilde{A}^{E}\right)=\phi\left(\Sigma^{E}\right)$ [17]. Thus $\operatorname{Ind}_{\phi}\left(\tilde{A}^{E}\right)=0$.

\section{Differential operator transversely elliptic to a Lie group action}

Let $G$ be a compact Lie group acting smoothly on a smooth compact manifold $V, E$ a complex hermitian $G$-vector bundle over $V$, and $P$ a classical pseudodifferential operator of order $m>0$ acting on the space of sections of $E$. We suppose first that $P$ is transversely elliptic, which means that the 
restriction of the principal symbol of $P$ to the cotangent bundle transverse to the action of $G$ is invertible, and otherwise that $P$ is quasi-invariant by $G$, which means that $\mathrm{gPg}^{-1}-P$ is of order strictly less than $m$.

We construct here a class in the analytical K-cohomology group $K^{*}(C(V) \times$ $\left.G):=K K^{*}(C(V) \rtimes G, \mathrm{C})\right)$, where $C(V) \rtimes G$ is the cross-product $\mathrm{C}^{*}$-algebra.

LEMma 9.1. With the notation above, for any $a \in C(V) \rtimes G$, the operator $a\left(1+P^{*} P\right)^{-1}$ is compact.

Proof. For the simplicity of the proof, we assume that the order of $P$ is one.

Let $\Delta_{G}$ be the Casimir invariant, an order 2 differential operator already considered in [2, ch2]. Then $P^{*} P+\Delta_{G}$ is an elliptic pseudodifferential operator, and thus has compact resolvent. Let $a$ be an element of $C_{c}^{\infty}(V \times G)$.

Let's show that $S=a\left(1+P^{*} P\right)^{-1}-a\left(1+P^{*} P+\Delta_{G}\right)^{-1}$ is compact. We have:

$$
\begin{aligned}
S= & a\left(1+P^{*} P+\Delta_{G}\right)^{-1} \Delta_{G}\left(1+P^{*} P\right)^{-1} \\
= & \left(1+P^{*} P+\Delta_{G}\right)^{-1} a \Delta_{G}\left(1+P^{*} P\right)^{-1} \\
& +\left[a,\left(1+P^{*} P++\Delta_{G}\right)^{-1}\right] \Delta_{G}\left(1+P^{*} P\right)^{-1} \\
= & \left(1+P^{*} P+\Delta_{G}\right)^{-1} a \Delta_{G}\left(1+P^{*} P\right)^{-1} \\
& +\left(1+P^{*} P+\Delta_{G}\right)^{-1}\left[a, P^{*} P+\Delta_{G}\right]\left(1+P^{*} P+\Delta_{G}\right)^{-1} \Delta_{G}\left(1+P^{*} P\right)^{-1}
\end{aligned}
$$

By classical Lie group analysis, the operator $a \Delta_{G}$ is bounded. Thus the first term in the last part of the equation is compact. The commutator is the second term is a equal to a first order pseudo-diffrential operator plus a bounded one, and thus its product by $\left(1+P^{*} P+\Delta_{G}\right)^{-1}$ is compact, and the whole expression too.

Thus $a\left(1+P^{*} P\right)^{-1}$ is compact for $a \in C_{c}^{\infty}(V \times G)$ and by continuity for all $a$ in the cross-product $C(V) \rtimes G$.

Proposition 9.2. With $P$ as above, the operator $F=P\left(1+P^{2}\right)^{-\frac{1}{2}}$ acting on $L^{2}(V, E)$ is a Fredholm module over $C(V) \rtimes G$.

Thus we obtain an element in $K_{*}(C(V) \times G)$. Now let $X$ be a locally compact topological $G$-space and $f: V \rightarrow X$ be a $G$-map. Then one gets a class $f_{*}([P]) \in K K(C(X) \rtimes G)$. as an immediate consequence of our main theorem, the latter is nul when it is a boundary:

Corollary 9.3. Let $Z$ be a $G$-manifold with boundary diffeomorphic to $V$, and suppose that $E$ extends to $\tilde{E}$ on $Z$, and let $Q$ be an pseudodifferential operator on $Z$, transversely elliptic and quasi-invariant under $G$, compatible 
with $P$, and suppose that $f: V \rightarrow X$ extends to $W$. Then the index class of $P$ in $K_{*}(C(X) \rtimes G)$ vanishes.

Proof. This follows from the fact that the triple $\left(L^{2}(V, E), P, \tau\right)$ is the boundary of $\left(L^{2}(Z, \tilde{E}), Q\right)$.

REMARK 9.4. A similar statement for the topological index has been obtained in a recent paper by C. Farsi [10]: a proof of the bordism invariance of the index map defined by M. F. Atiyah: $K_{G}^{0}\left(T_{G}^{*} V\right) \rightarrow \mathscr{D}^{\prime}(G)$ is carried out.

\section{Conservation of the index}

This section is devoted to a non-commutative version of a well known gluing theorem: let $V_{1}, V_{2}$ be two manifolds of dimension $n, M$ an $n-1$ compact manifold, $j_{i}: M \rightarrow V_{i}$ a separating embedding. Then one can split each $V_{i}$ along $j_{i}(M)$, intertwines the pieces and glue them to obtain two new manifolds $W_{1}$ and $W_{2}$. Suppose also that coefficients bundles are given, and consider Diract type operator twisted by those bundles. Then how is the sum of the indices of $V_{i}$ is related to that of $W_{i}$ ?

Such a theorem has been proved by U. Bunke [7] for (non-compact) Riemannian manifolds with scalar curvature uniformly positive at infinity, and for the index in value in the $\mathrm{K}$-theory of some $\mathrm{C}^{*}$-algebra. Let $\mathscr{A}, \mathscr{B}$ be two $\mathrm{C}^{*}$ algebras and $\mathscr{Z}=\left(\left(\mathscr{E}_{1}, T_{i}, \tau_{i}\right)\right)_{i=1, \ldots, n}$ be a finite sequence of Hilbert module with the same boundary $\left(\mathscr{E}_{b}, A\right)$. To this sequence we may associate an element of $K K(\mathscr{A}, \mathscr{B})$ as follows: let $z_{i} \in K K(\mathscr{A}, \mathscr{B})$ be the classe of $\mathscr{E}_{i} \sharp \tilde{\mathscr{E}}_{i+1}$ for $i \leq n-1$ and $z_{n}$ be that of $\mathscr{E}_{n} \sharp \tilde{\mathscr{E}}_{1}$, where $\tilde{\mathscr{E}}_{i}$ means the opposite Hilbert module. Then we show here that $z_{1}+\cdots+z_{n}$ is bordant to zero, and as a consequence of the main theorem, we get:

Proposition 10.1. With the previous hypothesis, one has $\sum_{i} z_{i}=0$ in the bivariant group $K K(\mathscr{A}, \mathscr{B})$.

Proof. Let $\mathscr{P}=\mathscr{E}_{b} \otimes L^{2}([0,1]) \otimes L^{2}(S)$, with $T=A \otimes 1 \otimes 1+1 \otimes$ $\partial_{1} \otimes 1+1 \otimes 1 \otimes \partial_{2}$, where $S$ is the circle of length $N+1$ covered by the interval $[0, n+1]$ by the map $x \rightarrow \exp \left(2 \pi \frac{x}{n+1}\right)$, and $\partial_{j}$ are the standard Dirac operators. Then we glue $\mathscr{E}_{i} \otimes L^{2}\left(\left[0, \frac{1}{2}\right]\right)$ on $\mathscr{P}$ by identifying the submodule $\mathscr{E}_{b} \otimes L^{2}\left(\left[0, \frac{1}{4}\right]\right) \otimes L^{2}\left(\left[k, k+\frac{1}{2}\right]\right)$ of $\mathscr{P}$ with the corresponding submodule of $\mathscr{E}_{i} \otimes L^{2}\left(\left[0, \frac{1}{2}\right]\right)$.

Then, as a result, one obtains a Hilbert module $(\tilde{\mathscr{P}}, \tilde{T}, \tilde{\tau})$ with boundary given by the direct sum of $\mathscr{E}_{1} \sharp \tilde{\mathscr{E}}_{2} \oplus \mathscr{E}_{2} \sharp \tilde{\mathscr{E}}_{3} \oplus \cdots \oplus \mathscr{E}_{n} \sharp \tilde{\mathscr{E}}_{1} \oplus\left(\mathscr{E}_{b} \otimes L^{2}(S)\right)$.

By Theorem 6.2, the corresponding element in $K K(\mathscr{A}, \mathscr{B})$ is zero, and as $\left[\mathscr{E}_{b} \otimes L^{2}(S)\right]=0$, the claimed is proven. 


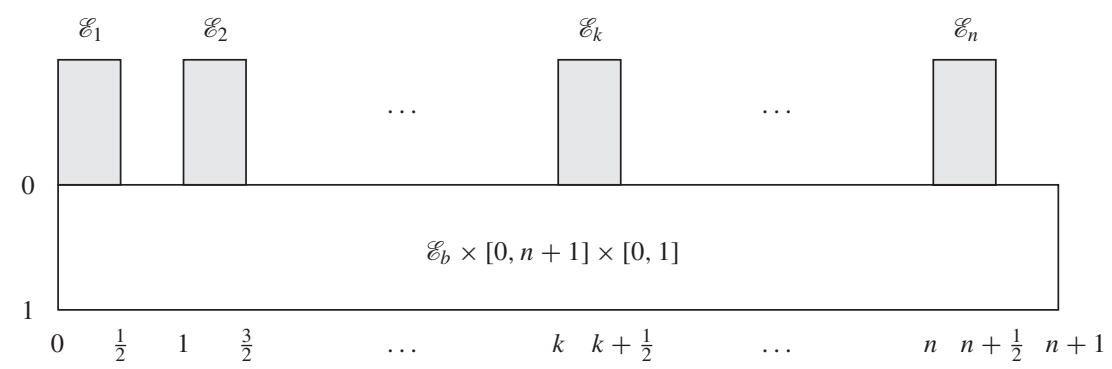

FIGURE 1. The Hilbert bimodule $\tilde{\mathscr{P}}$ (before identifying the end-sides)

A particular case is the following: let $\left(\mathscr{E}_{i, j}, T_{i, j}, \tau_{i, j}\right)$, for $i, j=1,2$, be Hilbert bimodules over $(\mathscr{A}, \mathscr{B})$ with boundary isomorphic to $\left(\mathscr{E}_{b}, A\right)$. Then, by using these isomorphims, we have four closed bimodules over $(\mathscr{A}, \mathscr{B})$, $\mathscr{P}_{1}=\mathscr{E}_{1,1} \# \mathscr{E}_{1,2}, \mathscr{P}_{2}=\mathscr{E}_{2,1} \# \mathscr{E}_{2,2}, \mathscr{Q}_{1}=\mathscr{E}_{2,1} \# \mathscr{E}_{1,2}, \mathscr{Q}_{2}=\mathscr{E}_{1,1} \# \mathscr{E}_{2,2}$, with corresponding operator $C_{1}, C_{2}, D_{1}, D_{2}$ and grading $\beta_{1}, \beta_{2}, \gamma_{1}, \gamma_{2}$, and one has classes in $K K(\mathscr{A}, \mathscr{B})$ simply denoted by $\left[\mathscr{P}_{1}\right]$ etc. Then:

Corollary 10.2. One has equality $\left[\mathscr{P}_{1}\right]+\left[\mathscr{P}_{2}\right]=\left[\mathscr{Q}_{1}\right]+\left[\mathscr{Q}_{2}\right]$ in $K K_{*}(\mathscr{A}, \mathscr{B})$.

PRoof. The claim follows from the previous proposition with $n=4$ and $\mathscr{E}_{1}=\mathscr{E}_{1,1}, \mathscr{E}_{2}=\mathscr{E}_{1,2}, \mathscr{E}_{3}=\mathscr{E}_{2,1}, \mathscr{E}_{4}=\mathscr{E}_{2,2}$

EXAMPLE 10.3. We shall retrieve the main theorem of Bettaieb, Matthey and Valette [5, Theorem 2.2]. Let as above $V_{1}, V_{2}$ be two manifolds of dimension $n, M$ an $n-1$ compact manifold, $j_{i}: M \rightarrow V_{i}$ a separating embedding. Then one can split each $V_{i}$ along $j_{i}(M)$, intertwine the pieces and glue them to obtain two new manifolds $W_{1}$ and $W_{2}$. Suppose that $f_{i}: V_{i} \rightarrow Z$ is a continuous map such that the restriction of $f_{1}$ and $f_{2}$ to $W$ (via $j_{i}$ ) are homotopic. Then the gluing gives canonically two maps $g_{i}: W_{i} \rightarrow Z$. Suppose next that there exist compatible $\operatorname{spin}^{c}$ structure on $V_{i}$ and $W_{i}$. Then the Dirac operator associated to the $\operatorname{spin}^{c}$ structure on $V_{i}\left(\right.$ resp. $\left.W_{i}\right)$ gives a class in $K_{0}\left(V_{i}\right)$ (resp. $\left.K_{0}\left(W_{i}\right)\right)$ and thus a class $\left[V_{i}\right]$ (resp. $\left.W_{i}\right)$ in $K_{0}(Z)$ by $f_{i, *}$ (resp. $\left.g_{i, *}\right)$. Then the last corollary reads as: $\left[V_{1}\right]+\left[V_{2}\right]=\left[W_{1}\right]+\left[W_{2}\right]$ in $K_{0}(Z)$.

\section{REFERENCES}

1. Atiyah, M. F., Patodi, V. K., and Singer, I. M., Spectral asymmetry and Riemannian geometry I, Math. Proc. Cambridge Philos. Soc. 77 (1975), 43-69.

2. Atiyah, M. F., Elliptic Operators and Compact Groups, Lecture Notes in Math. 401, Springer, Berlin 1974.

3. Baaj, S., and Julg, P., Théorie bivariante de Kasparov et operateurs non bornés dans les $C^{*}$-modules hilbertiens, C. R. Acad. Sci. Paris (I) 296 (1983), 875-878. 
4. Baum, P., Douglas, R. G., and Taylor, M. E., Cycles and relative cycles in analytic $K$ homology, J. Differential Geom. 30 (1989), 761-804.

5. Bettaieb, H., Matthey, M., and Valette, A., Unbounded symmetric operators in K-homology and the Baum-Connes conjecture, J. Funct. Anal. 229 (2005), 184-237.

6. Blackadar, B., $K$-theory for Operator Algebras. 2nd ed., Math. Sciences Research Institute Publ. 5, Cambridge Univ. Press, Cambridge 1998.

7. Bunke, U., A K-theoretic relative index theorem and Callias-type Dirac operators, Math. Ann. 303 (1995), 241-279.

8. Carvalho, C., A K-theory proof of the cobordism invariance of the index, K-Theory 36 (2005), $1-31$.

9. Carvalho, C., Cobordism invariance of the family index, preprint, 2008.

10. Farsi, C., Orbifold index cobordism invariance, Topology Appl. 156 (2007), 1770-1775.

11. Hilsum, M., Fonctorialité en K-théorie bivariante pour les variétés lipschitziennes, K-Theory 3 (1989), 401-440.

12. Hilsum, M., Index classes of hilbert modules with boundary, prépublication, Institut de Mathématiques de Jussieu 281 (2001).

13. Hilsum, M., Hilbert modules of foliated manifolds with boundary, pp. 315-332 in: Foliations: Geometry and Dynamics (Proc. Warsaw 2000), World Sci. Publ., River Edge, NJ 2002.

14. Lance, E. C., Hilbert $C^{*}$-modules, London Mathematical Society Lecture Note 210, Cambridge Univ. Press, Cambridge 1995.

15. Lück, W., and Schick, T., Various $L^{2}$-signatures and a topological $L^{2}$-signature theorem, pp. 362-399 in: High-dimensional Manifold Topology, World Sci. Publ., River Edge, NJ 2003.

16. Palais, R. S., Seminar on the Atiyah-Singer index theorem, Annals of Math. Studies 57, Princeton Univ. Press, Princeton 1965.

17. Rosenberg, J., and Weinberger, S., Higher G-signatures for Lipschitz manifolds, K-Theory 7 (1993), 101-132.

18. Teleman, N., The index theorem for topological manifolds, Acta Math. 153 (1984), 117-152.

C.N.R.S.

INSTITUT DE MATHÉMATIQUES DE JUSSIEU

175 RUE DU CHEVALERET

75013 PARIS

FRANCE

E-mail: hilsum@math.jussieu.fr 